\title{
Proceso interno de descentralización fiscal en el Distrito Capital 1992 1999
}

\author{
Teodomiro Muñoz Criollo*
}

Resumen. Los avances obtenidos sobre la descentralización, respaldados en la Constitución Política de 1991, doce años después están en retroceso, tanto por las reformas constitucionales posteriores como por las leyes que la han venido reglamentando. En el Distrito Capital, la Ley Primera de 1992 instauró las transferencias a las localidades con base en los ingresos totales del Distrito, con el fin de dar solución a las necesidades básicas insatisfechas de la población, luego el Estatuto Orgánico de Bogotá redujo el valor de las transferencias a un diez por ciento de los ingresos corrientes del Distrito, lo que ha imposibilitado el objetivo de las mismas. Se resalta el hecho de que las transferencias tienden a disminuir en los últimos años, observándose que existe una preponderancia centralista en el manejo de las transferencias y en la administración de las localidades, por lo tanto es este modelo el que más incide en la organización del territorio local. Palabras clave: Transferencias, transferencias por habitante, localidades, necesidades básicas y autonomía.

* Licenciado en Ciencias Sociales, Universidad Pedagógica Nacional. Magister en Geografía, EPG, Convenio UPTC-IGAC.

Perspectiva Geográfica 23 


\begin{abstract}
After twelve years of the 1991 Political Constitution, advances on decentralization which had already been reached and supported by it are now moving backwards. Not only due to the reforms which have been made afterwards, but also by the laws that have been regulating it. In the "Distrito Capital" (Bogotá) the first law, issued in 1992, established transferences (budget passing e.g. from the Nation to its different "Departamentos") to the districts based on the total incomes of the "Distrito" and aiming to find a solution to the basic, unsolved needs of the population. Later, the "Estatuto Orgánico" (political and administra tive organization system) of Bogotá reduced the transferences worth to only a ten per cent of the current incomes of the "Distrito". This fact has turned the original aim of the transferences impossible because their worth became excesively low, and in the past years its tendency has been to decrease steadily. It can be observed that there is a centralist trend both in the dealing with transferences and in the administration of the districts. Therefore it is this model which influences the most the organization of the local territory. Key words: transferences, transferences per inhabitant, districts, basic needs, and autonomy.
\end{abstract}

Sommaire. Les avances en décentralisation qui avaient été obtenues et soutenues lors de la Constitution Politique de 1991 sont en train de reculer douze ans après, d'un côté par les réformes constitutionelles ayant lieu après son issue, et de l'autre par les lois qui sont venues la réguler. Dans le "Distrito Capital "(Bogotá) la première loi, issue de 1992, a établi les transférences (budget qui passe p.ex. de la Nation aux differents Départements) aux arrondissements prenant comme base les recettes totales du "Distrito", à fin de chercher à résoudre les besoins primaires et insatisfaits de la population. Mais ensuite, le "Estatuto Orgánico" (système d'organisation politique et administrative) de Bogotá a rédui la valeur des transférences à un dix pour cent des recettes courantes du "Distrito", ce qui a troublé l'éfficacité de l'objectif des transférences, une fois que la valeur de celles ci est dévenue carrément basse et la tendence dans ces dernières annèes a montré qu'elle peut diminuer davantage. On peut donc noter qu'il y existe une prépondérance vis à vis du centralisme dans la façon dont les transférances sont manipulées et aussi dans ce qui concerne l'administration des arrondissements. Pour cette raison il est ce modèle ci qui a le plus d'influence dans l'organisation du territoire local. Mots clef: transférances, transférances par habitant, arrondis sements, besoins primaires et autonomie.

Aceptado: junio 2003.

24 Teodomiro Muñoz Criollo 


\section{Presentación}

La descentralización fiscal corresponde a la acción por medio de la cual el poder central del Estado transfiere recursos económicos a entidades territoriales, tales como departamentos distritos o municipios, para que éstos los administren de forma autónoma en planes o proyectos de desarrollo, que busquen satisfacer las necesidades básicas de la población. En este sentido se reconoce que la descentralización fiscal es un factor más dentro de todo el proceso de descentralización, el cual supone otros factores como la descentralización administrativa y la descentralización política, para que ésta se convierta en un proceso integral en la búsqueda de las soluciones de las necesidades básicas insatisfechas de la comunidad.

En el Distrito Capital, se vienen adelantando transferencias desde el año 1992, hacia sus veinte localidades en que está dividido internamente, de conformidad con la Constitución Política, la ley primera de 1992 y el Estatuto Orgánico de Bogotá.

\section{Antecedentes}

Los primeros intentos formales de transferencias en Bogotá se inician hacia el año 1972 mediante el Acuerdo 26 del mismo año. Dentro de los aspectos más importantes de éste acuerdo aparecen: la facultad a los Alcaldes Menores para celebrar contratos hasta por cien mil pesos y se crea por primera vez el situado presupuestal correspondiente al quince por ciento de los ingresos del Distrito, provenientes de los impuestos de industria y comercio, para ser destinados a gastos de funcionamiento de las Alcaldías Menores (Botero, 1990).

Sin embargo en la realidad los avances descentralizadores de este acuerdo no tuvieron aplicación como lo diagnosticó la "Comisión asesora para la reforma de regimenes de las Alcaldías Menores” (Botero, 1990).

En el año 1982 el Alcalde Mayor Augusto Ramírez Ocampo conformó la Comisión para la Descentralización, que elaboró un diagnóstico de la situación administrativa en las Alcaldías Menores, así como también propuso las siguientes recomendaciones que fueron tenidas en cuenta en el Acuerdo 8 de 1985: convertir al Alcalde Menor en ordenador del gasto, crear un Fondo Zonal supervisado por una Junta Administradora que se conformaría por el Alcalde Menor quien la preside, el Comandante de Policía de la zona, un representante de la comunidad, un delegado del Departamento de Planeación Distrital, un delegado de la Secretaría de Hacienda, un delegado de la Secretaría de Gobierno y por último, uno del Concejo de Bogotá. (Botero, 1990).

En 1987 se aprobó el Acuerdo 8 que establece la creación de las Juntas Administradoras Zonales y sus funciones, siendo las principales, la 
formulación del Plan de Desarrollo Local, la ratificación del Fondo de Desarrollo Zonal y las nuevas funciones administrativas del Alcalde Menor. Dentro del reglamento interno de las Juntas Administradoras se establece que éstas serán conformadas por seis miembros quienes deberán ser elegidos por voto directo entre los habitantes de la zona y poseerán las mismas inhabilidades que establece la ley para los concejales del Distrito Capital (Botero, 1990).

El Acuerdo 8 de 1987 fue uno de los avances formales más importantes dentro de los antecedentes que se presentaron con anterioridad a la Constitución Política de 1991, sin embargo, no logró entrar en vigencia, debido a que el Consejo de Estado declaró inexequible la creación de Juntas Administradoras Zonales en el Distrito Capital (Botero,1990).

\section{Proceso de descentra- lizacion fiscal en el Distrito Capital}

\subsection{Contexto socio-económico}

El proceso de descentralización fiscal en el Distrito Capital, se planteó dentro de un contexto en el cual las finanzas eran de gran preocupación, tanto por el crecimiento de la deuda pública como por el déficit fiscal (Sarmiento, 1995). Situación que fue asumida bajo la administración del Alcalde Mayor Jaime Castro a partir de tres estrategias a saber: primero, el esfuerzo fiscal realizado mediante la creación de nuevos impuestos a los contribuyentes principalmente en lo relacionado con industria y comercio, autoavalúo catastral y sobretasa a la gasolina, segunda, la venta de empresas distritales de servicios públicos y por último, el control a la evasión tributaria. Lo anterior ha permitido al Distrito estar tranquilo en materia de finanzas, puesto que se ha reducido la deuda hasta un nivel tolerable por los bancos prestamistas y el fisco distrital han llegado mostrar superávit (Sarmiento, 1995).

Sin embargo, estos logros en las finanzas del Distrito repercutieron en la ampliación de los gastos de funcionamiento al ensancharse la planta de personal que consumió, entre los años 1990 y 1993, el ochenta y dos por ciento en promedio de los ingresos corrientes, mientras que los gastos de inversión se descuidaron, generando un aumento en el deterioro de la calidad de vida de los bogotanos, que se evidenció en el aumento del porcentaje de pobreza, el cual pasó de $29.5 \%$ en el año 1986 a $38.1 \%$ en 1992 (Sarmiento, 1995).

\subsection{Inicios de la descentralización fiscal}

Con la promulgación de la Constitución Política de 1991 la descentralización avanza como propuesta administrativa del Estado; es así como con el Artículo Primero se

26 Teodomiro Muñoz Criollo 
propende por una forma de descentralización con autonomía de los entes territoriales que conforman la república. En cuanto a la descentralización fiscal la Constitución en el Artículo 356 establece el situado fiscal correspondiente a los ingresos corrientes de la Nación que se transfieren a los entes territoriales, con destinación específica en salud, educación y obras de infraestructura.

Para el Distrito Capital la Constitución Política, en su Articulo 322, le otorga el estatus de Distrito Especial aunque con la normatividad de un municipio, además de otros aspectos como la conformación del Concejo Municipal, la apropiación de las partidas correspondientes a las localidades por parte de las Juntas Administradoras Locales y la creación de los planes y programas de desarrollo integral.

El 28 de enero de 1992 se aprueba la Ley 01 por la cual se crean las Juntas Administradoras Locales en el Distrito Capital, en la cual se estableció que el $2.0 \%$ de los ingresos totales del presupuesto del Distrito Capital serian transferidos a las localidades, porcentaje que debería alcanzar como mínimo el veinte por ciento para el año 1998. Con base en esta ley se hicieron las primeras transferencias a las localidades en el año 1992. Pero con la aprobación del Estatuto Orgánico de Bogotá o Decreto 1421 de 1993 se modifica el porcentaje de las transferencias, fijándose en el diez por ciento de los ingresos corrientes de la tributación de los contribuyentes del
Distrito Capital, y no de los ingresos totales como lo establecía la Ley 01 de 1992.

Esto redundó en que el valor de las transferencias a las localidades disminuyera, y además que el incremento de estas, dependiera del incremento en los impuestos a los contribuyentes y no por el incremento de otras fuentes, como las privatizaciones o las transferencias Nacionales, como se presentó en el año 1999, cuando los ingresos totales del Distrito aumentaron a cuatro billones de pesos, por concepto de venta de activos de la Empresa de Teléfonos de Bogotá y la descapitalización de la Empresa de Energía de Bogotá. Sin embargo, las transferencias a las localidades sólo alcanzaron el $6.76 \%$ de los ingresos totales (ver figura 1).

En la misma figura también se puede observar que año a año las transferencias a las localidades van en aumento, sin embargo al utilizar el método de estandarización de las transferencias y deflactarlas al año 1993 de acuerdo con el Índice de Precios al Consumidor (IPC) establecido por Departamento Administrativo Nacional de Estadísticas (DANE), se observa que las transferencias aumentaron en el año 1996 durante la primera administración del Alcalde Antanas Mockus, pero en el año 1999 estas disminuyeron en la administración del Alcalde Enrique Peñalosa, pese a que fue un año en el que los ingresos totales del Distrito aumentaron (Ver figura 2). 


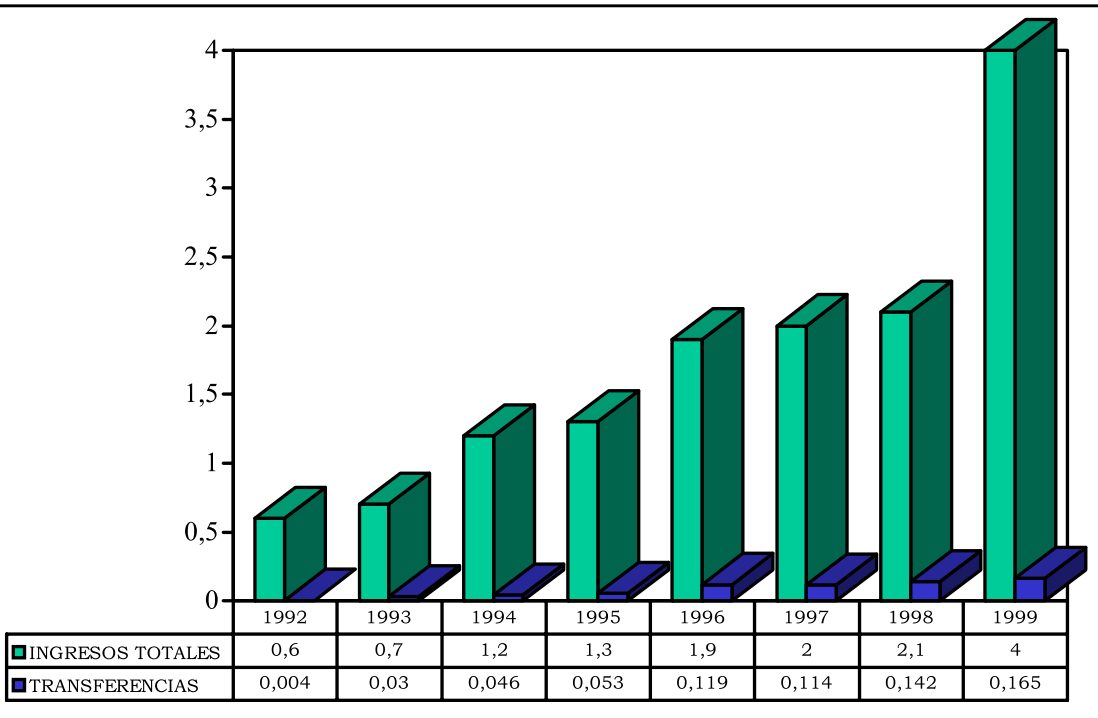

$\boldsymbol{\square I N G R E S O S ~ T O T A L E S ~} \boldsymbol{\square}$ TRANSEERENCIAS

Fuente: Saldías, C. 1999.

Figura 1

Evolución ingresos totales y transferencias a localidades 1992 - 1999

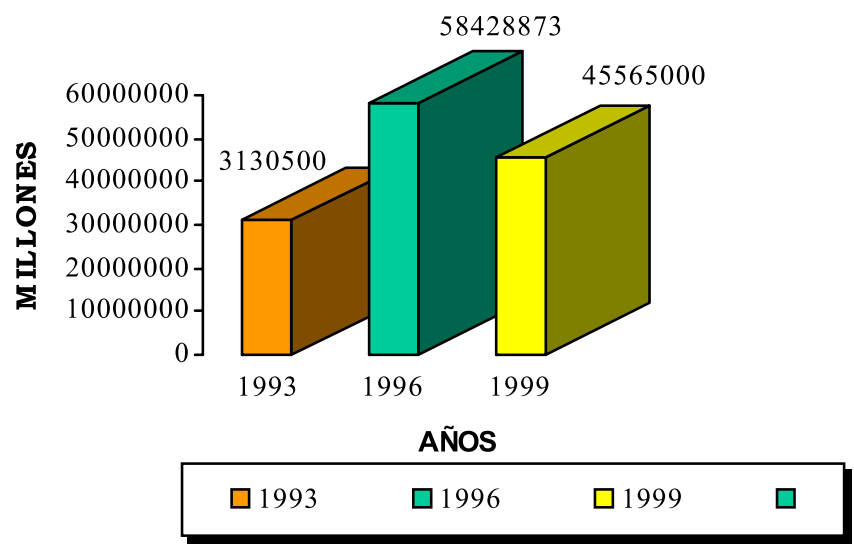

Fuente: Contraloría de Bogotá y cálculos del autor.

Figura 2

Transferencias estandarizadas en millones de pesos años 1993 - 1996 - 199

28 Teodomiro Muñoz Criollo 


\subsection{Fondos de desarrollo local}

Las transferencias que hace el Distrito a las localidades, son recepcionadas a través de los fondos de desarrollo local, que fueron credos para buscar soluciones a las necesidades básicas insatisfechas de la comunidad, mediante la inversión de las mismas. La asignación individual por localidad a partir del año 1994 se hizo con base en el indicador de necesidades básicas insatisfechas de la Encuesta sobre Pobreza y Calidad de Vida de diciembre de 1991 y el Censo de población y vivienda de 1993, con el objeto de hacer una distribución más equitativa de los recursos (Calderón, 1995).

Inicialmente los recursos se ejecutaban en las localidades mediante la presentación y aprobación de los proyectos incluidos en el Plan de Desarrollo Local que debía estar aprobado previamente por la Junta Administradora Local y firmado por el Alcalde Local. Esta situación cambió cuando en 1999 la Administración Distrital creó las Unidades Ejecutoras Locales (UEL), adscritas a las secretarías Distritales, para que aprobaran los proyectos de desarrollo.

Los proyectos de desarrollo se incorporan dentro de las prioridades y los sectores propuestos por el Plan de Desarrollo Distrital como guía para los planes de desarrollo local. Por ejemplo, en la administración Mockus las prioridades fueron: cultura ciudadana, medio ambiente, espacio público, progreso social, productividad urbana y legitimidad institucional. Los sectores fueron Promoción Social, Vivienda y Desarrollo Urbano, Salud, Educación, Cultura, Recreación y Deporte, Seguridad Ciudadana, Administración Local, Servicios Públicos, Transito, Transporte y Obras Viales, y por último, Apoyo Institucional y Concejo y Organismos de Control (Plan de Desarrollo Distrital. 1995- 1997).

\subsection{Evolución de los recursos asignados a los fondos de desarrollo local}

Las primeras asignaciones se hicieron en el año de 1992, ascendiendo a $\$ 4.337 .3$ millones y equivalían al $2 \%$ del total de los ingresos corrientes de la Administración Distrital, de los cuales sólo se ejecutaron $\$ 50.4$ millones, o sea el $1.16 \%$ del total de las transferencias. De la tabla 1 se deduce que en Chapinero, con una participación total de $\$ 120.178$ millones transferidos, se ejecutaron $\$ 24.157$ millones lo que equivale a $20.10 \%$; en Teusaquillo de 106.983 millones de pesos transferidos para este año, ejecutó 1.200 millones correspondientes al $1.2 \%$; en tanto que en La Candelaria de los $\$ 171.215$ millones transferidos se llevó a cabo una ejecución de $\$ 25.083$ millones correspondientes a $14.65 \%$. Las otras localidades no hicieron ejecuciones para este año. 
Tabla 1.

Trasferencias a las localidades 1992

\begin{tabular}{|c|l|c|c|c|}
\hline No. & LOCALIDAD & $\begin{array}{c}\text { PRESUPUESTO } \\
\text { EN MILLONES } \\
\text { DE PESOS }\end{array}$ & $\begin{array}{c}\text { EJECUCIÓN } \\
\text { EN MILLONES } \\
\text { DE PESOS }\end{array}$ & $\begin{array}{c}\text { EJECUCIÓN } \\
\%\end{array}$ \\
\hline 1 & USAQUEN & 127,355 & 0 & 0,0 \\
\hline 2 & CHAPINERO & 120,178 & 24,157 & 20.10. \\
\hline 3 & SANTA FE & 201,629 & 0 & 0 \\
\hline 4 & SAN CRISTOBAL & 325,321 & 0 & 0 \\
\hline 5 & USME & 305,173 & 0 & 0 \\
\hline 6 & TUNJUELITO & 204,703 & 0 & 0 \\
\hline 7 & BOSA & 247,174 & 0 & 0 \\
\hline 8 & KENNEDY & 275.280. & 0 & 0 \\
\hline 9 & FONTIBON & 210,977 & 0 & 0 \\
\hline 10 & ENGATIVA & 282,98 & 0 & 0 \\
\hline 11 & SUBA & 260,902 & 0 & 0 \\
\hline 12 & BARRIOS UNIDOS & 156,831 & 0 & 0 \\
\hline 13 & TEUSAQUILLO & 106,983 & 1.200 & 1,12 \\
\hline 14 & LOS MARTIRES & 133,68 & 0 & 0 \\
\hline 15 & ANTONIO NARIÑ & 186,311 & 0 & 0 \\
\hline 16 & PUENTE ARANDA & 119.300. & 0 & 0 \\
\hline 17 & LA CANDELARIA & 171,215 & 25,083 & 14,65 \\
\hline 18 & RAFAEL URIBE & 264,656 & 0 & 0 \\
\hline 19 & CIUDAD BOLIVAR & 409,788 & 0 & 0 \\
\hline 20 & SUMAPAZ & 216,865 & 0 & 0 \\
\hline & TOTAL & $\mathbf{4 . 3 3 7 . 3 0 1}$ & $\mathbf{5 0 . 4 4 0}$ & $\mathbf{1 , 1 6}$ \\
\hline
\end{tabular}

Fuente: Informe de la Contraloría de Bogotá D. C. 1992.

En 1993 se asignaron $\$ 30.317 .0$ millones de pesos y se ejecutaron \$26.125.0 millones, correspondientes al $86.2 \%$. Para este año todos los fondos locales hicieron ejecuciones presupuestales; se destacan Ciudad Bolívar con \$2.910,7 millones, San
Cristóbal con $\$ 2.407,9$ millones y Engativá con $\$ 2.087,4$. Sin embargo, el proceso de ejecución es bajo, corresponde a $86.2 \%$ en promedio, siendo Ciudad Bolívar la de menor ejecución con $77.3 \%$. 
De la tabla 2 se observa que el porcentaje de variación de las transferencias asignadas fue de $581 \%$; ello debido a que se pasa de 4.337 millones, en 1992 a 3.0317 millones de pesos, en 1993. El porcentaje de variación en la ejecución correspondió a $4071 \%$ dado que para este año todas las localidades hicieron ejecuciones en promedio del $86 \%$ mientras que en el año anterior solo tres localidades ejecutaron presupuesto de $1.16 \%$ en promedio. En la misma tabla se puede observar que las localidades con menor número de habitantes (La Candelaria, Antonio Nariño y Sumapaz) recibieron mayores transferencias, por encima de los doce mil pesos por habitante, mientras que las localidades con mayor número de población recibieron transferencias inferiores a los cuatro mil pesos por habitante (Ver mapa 1).

Para el año 1994 entra en vigencia el Estatuto Orgánico de Bogotá que establece que el $10 \%$ de los ingresos corrientes de la ciudad, menos los aportes de los establecimientos públicos y unidades de empresas industriales y comerciales, serán los aportes transferidos a los fondos locales. Ese mismo año se manejan aportes por un total de $\$ 46.379 .7$ millones de los cuales $\$ 40.391 .4$ millones corresponden a transferencias y lo demás corresponde al saldo del año anterior más recursos propios de las localidades (el Distrito tiene en cuenta para hacer las transferencias a las localidades el Censo de Población y Vivienda de 1993 y la Encuesta sobre Pobreza y Calidad de Vida). Como se observa en la tabla 3 el promedio de ejecución baja al $85,1 \%$, siendo las localidades con menor ejecución de proyectos: Antonio Nariño con $75.7 \%$, Ciudad Bolívar con $77.9 \%$ y Engativá con $67.6 \%$. La variación promedio en el porcentaje de presupuesto es de $53.0 \%$ dado que se pasa de $\$ 30.317$ millones a $\$ 46.379$ millones. Sin embargo, la ejecución en su variación pasa de $40.71 \%$ a $51.09 \%$; la razón por la cual se presenta este fenómeno es que todas las localidades están desarrollando ejecuciones presupuestales, pero a su vez todavía no cuentan con la suficiente experiencia ni infraestructura adecuada para hacer ejecuciones con mayor eficacia.

En 1995 la destinación presupuestal total fue de $\$ 53.942 .0$ millones, de los cuales se ejecutaron $\$ 37.893 .8$ millones equivalentes al $70.2 \%$ promedio. Siendo las localidades de Engativá con $19.2 \%$, Chapinero $26.0 \%$ y Tunjuelito con $55.0 \%$ las localidades con menor ejecución de proyectos de inversión y las de mayor inversión Bosa 93.1\%, Suba $90.8 \%$ y Rafael Uribe con $93.1 \%$. En promedio el porcentaje de variación en las transferencias aumenta en $16.31 \%$, mientras que su variación en ejecución disminuye pasando a $-4 \%$ (Ver tabla 4). 
Tabla 2

Transferencias a las localidades 1993

\begin{tabular}{|c|c|c|c|c|c|c|c|}
\hline No. & LOCALIDAD & $\begin{array}{c}\text { NÚMERO } \\
\text { DE } \\
\text { HABITANTES } \\
\end{array}$ & $\begin{array}{c}\text { PRESUPUESTO } \\
\text { POR } \\
\text { HABITANTE } \\
\end{array}$ & $\begin{array}{c}\text { PRESUPUESTO } \\
\text { EN MILLONES } \\
\text { DE PESOS } \\
\end{array}$ & $\begin{array}{c}\text { EJECUTADO } \\
\text { ENMILLONES } \\
\text { DE PESOS } \\
\end{array}$ & $\begin{array}{c}\text { VARIACIÓN } \\
1992-1993 \\
\text { ASIGNADO \% } \\
\end{array}$ & $\begin{array}{c}\text { VARIACIÓN } \\
\text { 1992-1993 } \\
\text { EJECUTADO \% } \\
\end{array}$ \\
\hline 1 & USAQUEN & 348.852 & 0,002875431 & 1003,10 & 843,1 & $688 \%$ & $843,10 \%$ \\
\hline 2 & CHAPINERO & 122.991 & 0,006272004 & 771,40 & 479,5 & $541 \%$ & $1884,93 \%$ \\
\hline 3 & SANTAFE & 102.244 & 0,014071241 & 1438,70 & 1.260 .4 & $614 \%$ & $1260,40 \%$ \\
\hline 4 & SAN CRISTOBAL & 439.559 & 0,00547799 & 2407,90 & 1.640 .3 & $640 \%$ & $1640,30 \%$ \\
\hline 5 & USME & 200.892 & 0,010444916 & 2098,30 & 2.064 .3 & $588 \%$ & $2064,30 \%$ \\
\hline 6 & TUNJUELITO & 204.367 & 0,006982047 & 1426,90 & 1.302 .0 & $597 \%$ & $1302,00 \%$ \\
\hline 7 & BOSA & 215.816 & 0,00799709 & 1725,90 & 1.587 .3 & $598 \%$ & $1587,30 \%$ \\
\hline 8 & KENNEDY & 758.870 & 0,000161029 & 122,20 & 1.723 .3 & $577 \%$ & $1723,30 \%$ \\
\hline 9 & FONTIBON & 201.610 & 0,007282377 & 1468,20 & 1.460 .4 & $596 \%$ & $1460,40 \%$ \\
\hline 10 & ENGATIVA & 671.360 & 0,000428086 & 287,40 & 1.796 .2 & $638 \%$ & $1796,20 \%$ \\
\hline 11 & SUBA & 564.658 & 0,003243025 & 1831,20 & 1.188 .9 & $602 \%$ & $1188,90 \%$ \\
\hline 12 & B. UNIDOS & 176.552 & 0,006208936 & 1096,20 & 1.038 .8 & $599 \%$ & $1083,80 \%$ \\
\hline 13 & TEUSAQUILLO & 125.125 & 0,005596004 & 700,20 & 662,1 & $554 \%$ & $55075 \%$ \\
\hline 14 & MARTIRES & 95.541 & 0,009788468 & 935,20 & 873,2 & $600 \%$ & $873,20 \%$ \\
\hline 15 & A. NARIÑO & 98.355 & 0,01263586 & 1242,80 & 1.110 .4 & $567 \%$ & $1110,40 \%$ \\
\hline 16 & P. ARANDA & 282.481 & 0,003076313 & 869,00 & 849,1 & $628 \%$ & $849,10 \%$ \\
\hline 17 & CANDELARIA & 27.450 & 0,041446266 & 1137,70 & 978,1 & $564 \%$ & $471,27 \%$ \\
\hline 18 & RAFAEL URIBE & 379.259 & 0,005124466 & 1943,50 & 1.720 .4 & $608 \%$ & $1720,40 \%$ \\
\hline 19 & CIUDAD BOLIVAR & 418.609 & 0,006953267 & 2910,70 & 2.250 .0 & $610 \%$ & $2250,00 \%$ \\
\hline 20 & SUMAPAZ & 8.413 & 0,154665399 & 1301,20 & 1.252 .2 & $218 \%$ & $1252,20 \%$ \\
\hline & TOTAL & 5'443.004 & 0,005570031 & 30317,70 & 26.125 .0 & $581 \%$ & $4071,82 \%$ \\
\hline
\end{tabular}

Fuente: Informe de la contraloría de Bogotá D.C. 1993.Censo DANE. 1993 y Cálculos del autor. 
Tabla 3

Trasferencias a las localidades 1994

\begin{tabular}{|c|c|c|c|c|c|c|c|c|}
\hline No. & LOCALIDAD & $\begin{array}{c}\text { NÚMERO } \\
\text { DE } \\
\text { HABITANTES } \\
\end{array}$ & $\begin{array}{c}\text { TRANSFERENCIAS } \\
\text { POR } \\
\text { HABITANTE } \\
\end{array}$ & $\begin{array}{c}\text { PRESUPUESTO } \\
\text { ENMILLONES } \\
\text { DE PESOS } \\
\end{array}$ & $\begin{array}{l}\text { EJECUTADO } \\
\text { ENMILLONES } \\
\text { DE PESOS } \\
\end{array}$ & $\begin{array}{c}\text { EJECUCIÓN } \\
\text { EN } \\
\% \\
\end{array}$ & $\begin{array}{c}\text { VARIACIÓN } \\
\text { 1993-1994 } \\
\text { PARESUP. \% } \\
\end{array}$ & $\begin{array}{c}\text { VARIACIÓN } \\
1993-1994 \\
\text { EJECUTADO \% }\end{array}$ \\
\hline 1 & USAQUEN & 370.326 & 0,005908848 & 2188,2 & 1.970 .0 & 90 & $118,14 \%$ & $133,66 \%$ \\
\hline 3 & SANTAFE & 100.145 & 0,02222078 & 2225,3 & 2.178 .6 & 97,9 & $54,67 \%$ & $72,85 \%$ \\
\hline 4 & SANCRISTOBAL & 453.071 & 0,008342622 & 3779,8 & 3.254 .9 & 86,1 & $56,97 \%$ & $98,43 \%$ \\
\hline 7 & BOSA & 231.591 & 0,010481841 & 2427,5 & 2.362 .3 & 97,3 & $40,65 \%$ & $48,83 \%$ \\
\hline 8 & KENNEDY & 787.951 & 0,003748837 & 2953,9 & 2.494 .2 & 84,4 & $53,67 \%$ & $44,73 \%$ \\
\hline 9 & FONTIBON & 206.969 & 0,009245346 & 1913,5 & 1.485 .3 & 77,6 & $30,33 \%$ & $1,71 \%$ \\
\hline 10 & ENGATIVA & 691.397 & 0,006383308 & 4413,4 & 2.981 .7 & 67,6 & $111,43 \%$ & $66,00 \%$ \\
\hline 15 & ANTONIO NARIÑO & 96.852 & 0,016866972 & 1633,6 & 1.236 .0 & 75,7 & $31,45 \%$ & $11,31 \%$ \\
\hline 16 & PUENTE ARANDA & 279.771 & 0,00460734 & 1289 & 1.196 .1 & 92,8 & $48,33 \%$ & $40,87 \%$ \\
\hline 17 & CANDELARIA & 27.041 & 0,057216819 & 1547,2 & 1.312 .8 & 84,9 & $35,99 \%$ & $34,22 \%$ \\
\hline 18 & RAFAEL URIBE & 403.026 & 0,008663461 & 3491,6 & 2.954 .8 & 84,6 & $79,66 \%$ & $71,75 \%$ \\
\hline 19 & CIUDAD BOLIVAR & 431.879 & 0,010108387 & 4365,6 & 3.402 .5 & 77,9 & $49,98 \%$ & $51,22 \%$ \\
\hline 20 & SUMAPAZ & 8.643 & 0,202880944 & 1753,5 & 1.686 .7 & 96,2 & $34,76 \%$ & $34,70 \%$ \\
\hline & TOTALES & $5,602.210$ & 0,008278822 & 46379,7 & 39.471 .0 & 85,1 & $52,98 \%$ & $51,09 \%$ \\
\hline
\end{tabular}

Fuente: Informe de la Contraloría de Bogotá D.C. 1994 y Cálculos del autor. 
Tabla 4

Transferencias a las localidades 1995

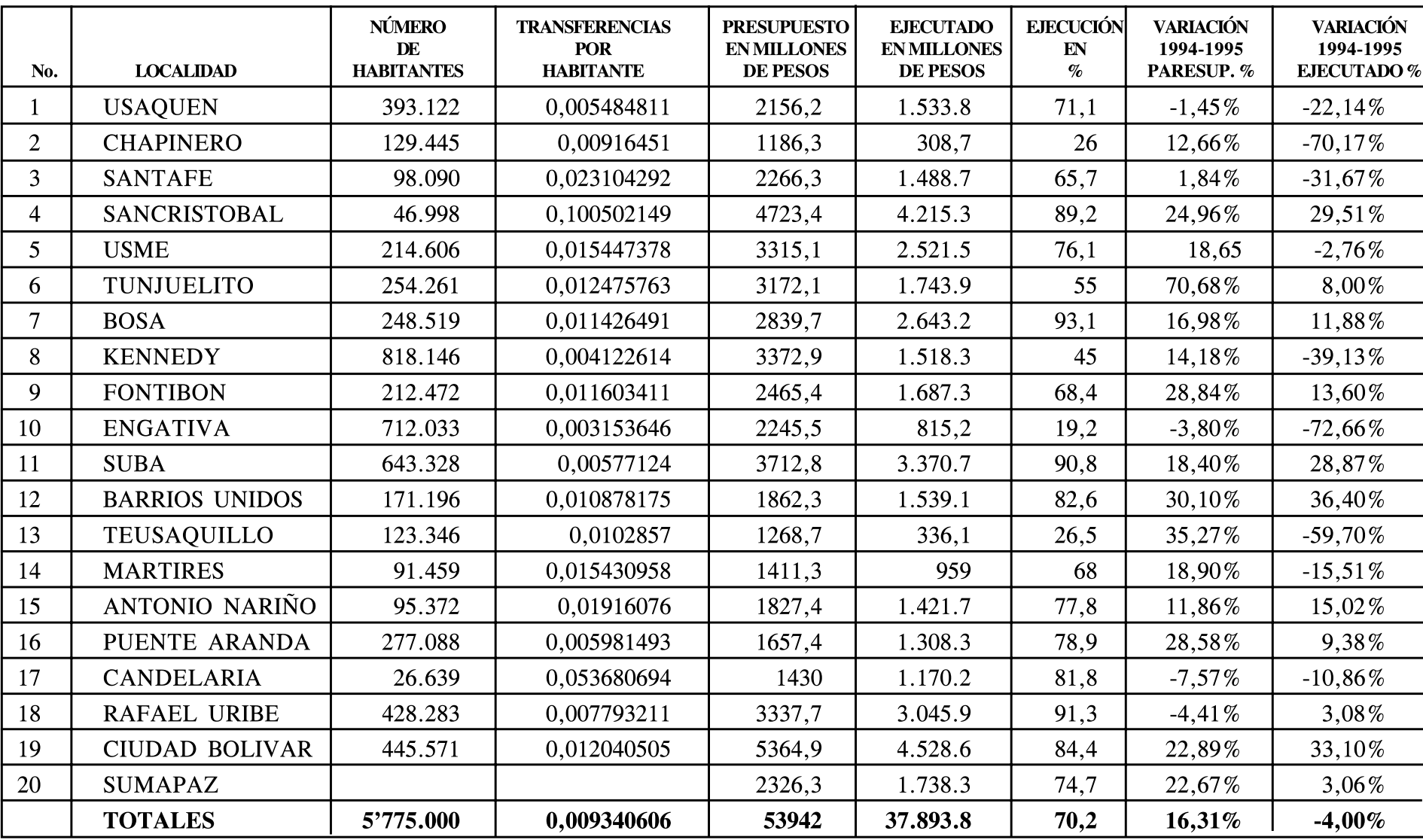

Fuente: Informe de la Contraloría de Bogotá D.C. y cálculos del autor. 
En 1996 se presenta una variación muy importante en las transferencias por cuanto pasa de $\$ 53.942 .0$ millones a $\$ 119.444 .0$ millones que corresponden $121.4 \%$ en los recursos asignados; sin embargo, las localidades no aprovecharon en su totalidad estos recursos, puesto que el promedio de la ejecución bajo a $54.50 \%$ de lo asignado. Se destaca que en la localidad de Engativá la variación del presupuesto fue de $513 \%$, seguida de Fontibón con $270.76 \%$. Todas las localidades presentan índices positivos de variación, siendo Suba la de menor variación con $77.63 \%$, denotándose la problemática constante de la ineficacia en la ejecución principalmente en el año que se aprueban los proyectos. Las localidades que recibieron mayores aportes fueron Engativá con $\$ 13.765 .6$ millones y Ciudad Bolívar con $\$ 11.953 .9$ millones, mientras que Teusaquillo tuvo $\$ 2.404 .6$ millones y Chapinero $\$ 2.664 .6$ millones fueron las que menos aportes recibieron (Ver tabla 5).

En la tabla 6 se muestran las transferencias para el año de 1997. En todas las localidades éstas se redujeron a $\$ 114.681 .5$ millones, presentando una variación de $-13.74 \%$; sin embargo, este año fue el de mayor ejecución del presupuesto con un promedio de $88.3 \%$ y una variación en relación con el año anterior de $58.17 \%$. Se destacan Chapinero con 98.9\% equivalente a $\$ 2074.8$ millones, Engativá y Ciudad Bolívar continúan siendo las de mayor asignación presupuestal con
$\$ 14.120 .7$ y $\$ 11.503 .3$ millones respectivamente.

Las transferencias calculadas correspondientes al año de 1998 ascendían a $\$ 120.886 .5$ millones destinados a los veinte fondos existentes, sin embargo se transfirieron $\$ 120.847 .2$ millones, presentándose una diferencia no sustentada de 39.3 millones. Esta cifra fue inferior al $10 \%$ establecida en el Estatuto Orgánico, pero posteriormente se hicieron nuevas adiciones presupuéstales con giros por $\$ 26.687 .3$ millones, por lo tanto, al concluir la vigencia se totalizó una apropiación definitiva por $\$ 142.232,8$ millones. En la tabla 7 se observa para este año el comportamiento del presupuesto por localidad y el presupuesto por habitante.

El último año del cual se ha podido obtener información sobre transferencias a las localidades es 1999; para este año ascendieron a 165.412 millones de pesos. Las localidades que mayor presupuesto recibieron fueron Ciudad Bolívar con $\$ 17.731$ millones, seguida de San Cristóbal con $\$ 15.241$ millones, Engativá con \$14.988.millones, Suba con $\$ 11.777$ millones y Kennedy con $\$ 11.733$ millones de pesos. Mientras que las localidades con menores asignaciones presupuestales fueron $\mathrm{La}$ Candelaria con $\$ 1.604$ millones y Los Mártires con $\$ 2.847$ millones. Se observa una gran eficiencia en las ejecuciones del presupuesto, la cual 
Tabla 5

Transferencias a las localidades 1996

\begin{tabular}{|c|c|c|c|c|c|c|c|c|}
\hline No. & LOCALIDAD & $\begin{array}{c}\text { NÚMERO } \\
\text { DE } \\
\text { HABITANTES }\end{array}$ & $\begin{array}{c}\text { TRANSFERENCIAS } \\
\text { POR } \\
\text { HABITANTE }\end{array}$ & $\begin{array}{l}\text { PRESUPUESTO } \\
\text { ENMILLONES } \\
\text { DE PESOS }\end{array}$ & $\begin{array}{l}\text { EJECUTADO } \\
\text { ENMILLONES } \\
\text { DE PESOS }\end{array}$ & $\begin{array}{c}\text { EJECUCIÓN } \\
\text { EN } \\
\%\end{array}$ & $\begin{array}{l}\text { VARIACIÓN } \\
\text { 1995-1996 } \\
\text { PARESUP. \% }\end{array}$ & $\begin{array}{c}\text { VARIACIÓN } \\
\text { 1995-1996 } \\
\text { EJECUTADO \% }\end{array}$ \\
\hline 1 & USAQUEN & 417.321 & 0,013984199 & 5835,9 & 2.742 .2 & $47,00 \%$ & $170,00 \%$ & $78,78 \%$ \\
\hline 3 & SANTAFE & 96.077 & 0,053944232 & 5182,8 & 1.977 .8 & $38,20 \%$ & $128,66 \%$ & $32,85 \%$ \\
\hline 4 & SANCRISTOBAL & 481.354 & 0,019563564 & 9417 & 5.772 .2 & $61,50 \%$ & $99,36 \%$ & $36,93 \%$ \\
\hline 7 & BOSA & 266.685 & 0,019663273 & 5243,9 & 1.842 .9 & $35,10 \%$ & $84,66 \%$ & $-30,27 \%$ \\
\hline 8 & KENNEDY & 849.499 & 0,011067111 & 9401,5 & 4.894 .2 & $52,10 \%$ & $178,73 \%$ & $222,34 \%$ \\
\hline 9 & FONTIBON & 218.121 & 0,025265793 & 5511 & 3.138 .2 & $56,90 \%$ & $270,76 \%$ & $85,98 \%$ \\
\hline 10 & ENGATIVA & 733.285 & 0,01877251 & 13765,6 & 5.441 .2 & $39,50 \%$ & $513,00 \%$ & $567,46 \%$ \\
\hline 15 & ANTONIO NARIÑO & 93.915 & 0,037580791 & 3529,4 & 1.702 .3 & $48,20 \%$ & $93,13 \%$ & $19,73 \%$ \\
\hline 16 & PUENTE ARANDA & 274.430 & 0,013533141 & 3713,9 & 2.504 .6 & $67,40 \%$ & $124,07 \%$ & $91,43 \%$ \\
\hline 17 & CANDELARIA & 26.242 & 0,104382288 & 2739,2 & 1.564 .4 & $57,10 \%$ & $91,55 \%$ & $33,68 \%$ \\
\hline 18 & RAFAEL URIBE & 455.123 & 0,015678838 & 7135,8 & 4.020 .9 & $56,30 \%$ & $113,79 \%$ & $32,01 \%$ \\
\hline 19 & CIUDAD BOLIVAR & 459.696 & 0,026003924 & 11953,9 & 7.513 .6 & $62,90 \%$ & $122,80 \%$ & $65,91 \%$ \\
\hline 20 & SUMAPAZ & & & 4796,1 & 2.773 .5 & $57,80 \%$ & $113,90 \%$ & $59,55 \%$ \\
\hline & TOTALES & $5,953.118$ & 0,020064108 & 119444 & 65.081 .8 & $54,50 \%$ & $121,43 \%$ & $71,74 \%$ \\
\hline
\end{tabular}

Fuente: Informe de la contraloría de Bogotá D.C. y Cálculos del autor. 
Tabla 6

Transferencias a las localidades 1997

\begin{tabular}{|c|c|c|c|c|c|c|c|c|}
\hline No. & LOCALIDAD & $\begin{array}{c}\text { NÚMERO } \\
\text { DE } \\
\text { HABITANTES }\end{array}$ & $\begin{array}{c}\text { TRANSFERENCIAS } \\
\text { POR } \\
\text { HABITANTE } \\
\end{array}$ & $\begin{array}{c}\text { PRESUPUESTO } \\
\text { ENMILLONES } \\
\text { DE PESOS } \\
\end{array}$ & $\begin{array}{c}\text { EJECUTADO } \\
\text { ENMILLONES } \\
\text { DE PESOS }\end{array}$ & $\begin{array}{c}\text { EJECUCIÓN } \\
\text { EN } \\
\% \\
\end{array}$ & $\begin{array}{c}\text { VARIACIÓN } \\
\text { 1995-1996 } \\
\text { PARESUP. \% }\end{array}$ & $\begin{array}{c}\text { VARIACIÓN } \\
1995-1996 \\
\text { EJECUTADO \% }\end{array}$ \\
\hline 1 & USAQUEN & 443.010 & 0,013073746 & 5791,8 & 5.143 .2 & 88,8 & $-0,75 \%$ & $87,55 \%$ \\
\hline 3 & SANTAFE & 94.105 & 0,049425642 & 4651,2 & 3.051 .2 & 65,6 & $-10,25 \%$ & $55,27 \%$ \\
\hline 4 & SANCRISTOBAL & 496.150 & 0,020839867 & 10339,7 & 9.574 .6 & 92,6 & $9,79 \%$ & $65,87 \%$ \\
\hline 7 & BOSA & 286.179 & 0,023940261 & 6851,2 & 6.590 .9 & 96,2 & $25,68 \%$ & $257,63 \%$ \\
\hline 8 & KENNEDY & 882.053 & 0,007592741 & 6697,2 & 6.268 .6 & 93,6 & $-33,32 \%$ & $28,08 \%$ \\
\hline 9 & FONTIBON & 223.920 & 0,021784566 & 4878 & 3.902 .4 & 80 & $-29,18 \%$ & $24,35 \%$ \\
\hline 10 & ENGATIVA & 755.171 & 0,018698679 & 14120,7 & 12.186 .2 & 86,3 & $-11,47 \%$ & $123,96 \%$ \\
\hline 11 & SUBA & 733.416 & 0,009960514 & 7305,2 & 6.910 .8 & 94,6 & $4,78 \%$ & $36,92 \%$ \\
\hline 15 & ANTONIO NARIÑO & 92.480 & 0,037125865 & 3433,4 & 3.083 .2 & 89,8 & $-12,64 \%$ & $81,11 \%$ \\
\hline 16 & PUENTE ARANDA & 271.798 & 0,014001575 & 3805,6 & 3.474 .6 & 91,3 & $-6,44 \%$ & $38,72 \%$ \\
\hline 17 & CANDELARIA & 25.852 & 0,072601733 & 1876,9 & 1.736 .2 & 92,5 & $-36,61 \%$ & $10,98 \%$ \\
\hline 18 & RAFAEL URIBE & 483.645 & 0,01435495 & 6942,7 & 6.123 .5 & 88,2 & $-14,18 \%$ & $52,29 \%$ \\
\hline 19 & CIUDAD BOLIVAR & 474.269 & 0,023932199 & 11350,3 & 9.398 .1 & 82,8 & $-21,30 \%$ & $25,08 \%$ \\
\hline 20 & SUMAPAZ & & & 4672,3 & 3.443 .5 & 73,7 & $-28,20 \%$ & $24,15 \%$ \\
\hline & TOTALES & 6'136.731 & 0,000189201 & 114681,5 & 101.263 .8 & 88,3 & $-13,74 \%$ & $58,17 \%$ \\
\hline
\end{tabular}

Fuente: Informe de la Contraloría de Bogotá D.C. 1993 y Cálculos del autor. 
Tabla 7

Transferencias a las localidades 1998

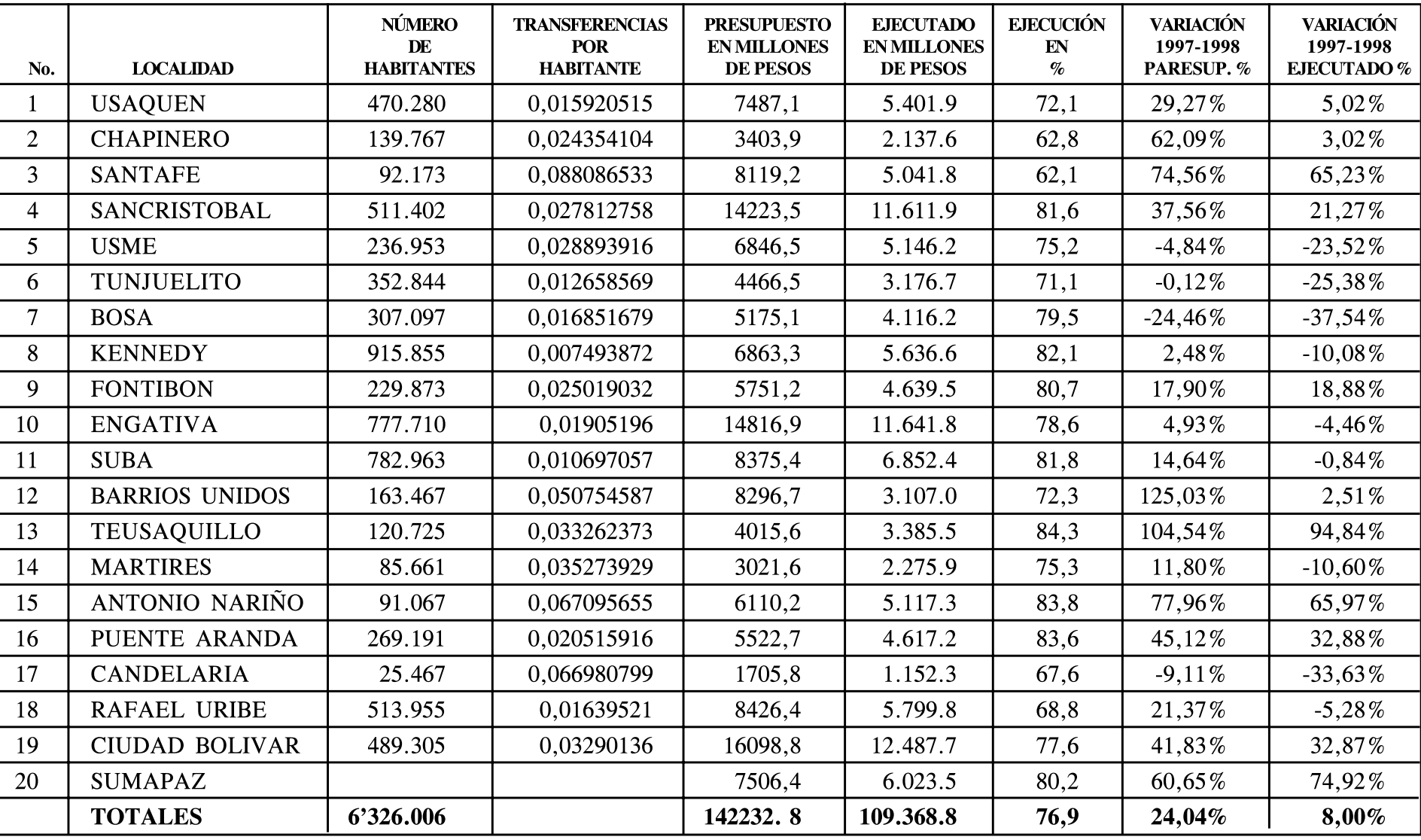

Fuente: Informe de la contraloría de Bogotá D.C. y Cálculos del autor. 
Tabla 8

Transferencias a las localidades 1999

\begin{tabular}{|c|l|c|c|c|c|c|}
\hline No. & \multicolumn{1}{|c|}{ LOCALIDAD } & $\begin{array}{c}\text { PRESUPUESTO } \\
\text { ENMILONES } \\
\text { DE PESOS }\end{array}$ & $\begin{array}{c}\text { EJECUTADO } \\
\text { EN } \\
\text { DE PESONES }\end{array}$ & $\begin{array}{c}\text { EJECUCIÓN } \\
\text { EN } \\
\mathbf{\%}\end{array}$ & $\begin{array}{c}\text { VARIACIÓN } \\
\mathbf{1 9 9 7 - 1 9 9 8} \\
\text { PARESUP.\% }\end{array}$ & $\begin{array}{c}\text { VARIACIÓN } \\
\text { 1997-1998 } \\
\text { EJECUTADO\% }\end{array}$ \\
\hline 1 & USAQUEN & $\mathbf{7 5 8 8 , 1}$ & 7833 & 103,23 & 1,35 & 0,01 \\
\hline 2 & CHAPINERO & 4371,5 & 4366,6 & 99,89 & 28,43 & 0,28 \\
\hline 3 & SANTAFE & 7972,6 & 8136,7 & 102,06 & $-1,81$ & $-0,02$ \\
\hline 4 & SANCRISTOBAL & 15241,1 & 15307,5 & 100,44 & 7,15 & 0,07 \\
\hline 5 & USME & 8403,2 & 8122 & 96,65 & 22,74 & 0,23 \\
\hline 6 & TUNJUELITO & 5801,8 & 5766,1 & 99,38 & 29,90 & 0,30 \\
\hline 7 & BOSA & 9303,7 & 9341,4 & 100,41 & 79,78 & 0,80 \\
\hline 8 & KENNEDY & 11733,4 & 11812,3 & 100,67 & 70,96 & 0,71 \\
\hline 9 & FONTIBON & 5777,7 & 5797,9 & 100,35 & 0,46 & 0,00 \\
\hline 10 & ENGATIVA & 14988,1 & 14488,1 & 96,66 & 1,16 & 0,01 \\
\hline 11 & SUBA & 11777,7 & 11841,9 & 100,55 & 40,62 & 0,41 \\
\hline 12 & BARRIOS UNIDOS & 4643,6 & 4626,5 & 99,63 & $-44,03$ & $-0,44$ \\
\hline 13 & TEUSAQUILLO & 4121,3 & 4149 & 100,67 & 2,63 & 0,03 \\
\hline 14 & MARTIRES & 2847 & 2915,5 & 102,41 & $-5,78$ & $-0,06$ \\
\hline 15 & ANTONIO NARIÑO & 3825 & 4123,7 & 107,81 & $-37,40$ & $-0,37$ \\
\hline 16 & PUENTE ARANDA & 6982,4 & 6966,1 & 99,77 & 26,43 & 0,26 \\
\hline 17 & CANDELARIA & 1604 & 1675,9 & 104,48 & $-5,97$ & $-0,06$ \\
\hline 18 & RAFAEL URIBE & 12154,4 & 12185,3 & 100,25 & 44,24 & 0,44 \\
\hline 19 & CIUDAD BOLIVAR & 17731,6 & 18363,9 & 103,57 & 10,14 & 0,10 \\
\hline 20 & SUMAPAZ & 8543,9 & 8180 & 95,74 & 13,82 & 0,14 \\
\hline & TOTALES & $\mathbf{1 6 5 4 1 2 , 1}$ & $\mathbf{1 6 5 9 9 9 , 4}$ & $\mathbf{1 0 0 , 3 6}$ & & \\
\hline
\end{tabular}

Fuente: Informe de la Contraloría de Bogotá D.C. y cálculos del autor. 
obtuvo el 100.4\%; en localidades como Antonio Nariño alcanzó el $107.8 \%$ y en La Candelaria con $104.5 \%$, en ambos casos superiores al promedio total (Ver tabla 8).

\subsection{Transferencias a las localidades en 1996 y 1999 estandarizadas a 1993}

Continuando con el método de estandarización propuesto por el DANE con base en el IPC, para el año 1996 el valor total da las transferencias aumentó en relación con 1993, situación que permitió a todas las localidades recibir transferencias por habitante superiores a los cuatromil pesos y en localidades con serias necesidades básicas como Usme y Ciudad Bolívar, las transferencias ascendieron a docemil pesos por habitante, como se evidencia en el mapa 2. Sin embargo el valor de las transferencias por habitante no es suficiente para la destinación con que fueron creadas.

En 1999 el valor de las transferencias baja y su distribución en las localidades también disminuye, tanto que a excepción de la localidad de Sumapáz, todas las localidades reciben transferencias por habitante inferiores a cuatro mil pesos por habitante (mapa 3).

Se suma a lo anterior la creación de las Unidades Ejecutoras Locales, Adscritas a las secretarías Distritales, que generaron un trámite más y restaron autonomía a las localidades en la destinación del presupuesto, dejando al arbitrio de las secretarías la tramitación de los recursos. ( Saldias, 1999).

\subsection{Transferencias por rubros en las localidades}

Los rubros prioritarios en los que las localidades han invertido las transferencias buscan estar en concordancia con la satisfacción de sus necesidades básicas. Tales rubros se destinan conforme lo establecen los planes de desarrollo locales agrupándolos en las siguientes categorías:

- Gastos de funcionamiento. Este rubro corresponde al salario de los ediles; a la dotación de materiales y suministros para el adecuado funcionamiento de la alcaldía, el fondo de desarrollo local y la junta administradora local; y al establecimiento de elementos de logística para la seguridad ciudadana de la localidad.

- Vías y transporte. Que corresponde a la construcción de nuevas vías, mantenimiento de las mismas, pavimentación vial, recuperación de la malla vial, espacio público, programa de señalización, restitución de andenes.

- Desarrollo educativo. Enfocado hacia el mejoramiento de la educación a través de la construcción

40 Teodomiro Muñoz Criollo 
de obras de infraestructura en los planteles, pago de alquiler a particulares por arrendamientos de instalaciones educativas, compra de terrenos para la construcción de nuevos colegios, dotación de materiales educativos a las instituciones, capacitación a la comunidad, campañas educativas, recreativas y culturales.

- Servicios básicos. Las principales inversiones en este rubro corresponden a la construcción y mantenimiento de las redes de alcantarillado, campañas de vacunación, brigadas de aseo y salud, asistencia social, construcción de centros comunitarios, encerramiento y adecuación de parques y zonas verdes. Concentrando el presupuesto en la población con más altos índices de N.B.I. de la localidad.

- Medio ambiente. Dedicado a reorientar los procesos de deterioro ambiental y ejecución de proyectos que disminuyan, controlen y prevengan el desmejoramiento de la calidad del entorno en la localidad con programas de reciclaje, limpieza de las rondas de los ríos y canales y campañas de arborización.
- Desarrollo rural. Enfocado a la capacitación y apoyo al campesino mediante asesorías en manejo de cultivos, eficiencia en organización de fincas, construcción de vías y comunicación en el área rural.

El rubro de gastos de funcionamiento aumenta constantemente, lo cual es preocupante, porque disminuye los gastos de inversión en los demás. Además, está contraviniendo el Estatuto Orgánico, puesto que en éste se señala que las transferencias sólo se deben utilizar para inversión y no para funcionamiento. El rubro de medio ambiente es al que menos importancia le han otorgado las localidades durante los tres períodos que se observaron, con excepción de las localidades de Fontibón y Engativá, en donde si bien no ha sido tan elevado el porcentaje de inversión por lo menos ha sido constante (ver mapa 4).

Finalmente se resalta el hecho de que los alcances de las transferencias a las localidades no permiten el desarrollar proyectos que propendan por una calidad de vida digna. También, el bajo poder administrativo de las instituciones locales son reflejo de que la mayor incidencia en la organización de los territorios locales se sigue realizando bajo los esquemas administrativos centralistas del Estado. 


\section{Bibliografía}

ALCALDIA MAYOR DE SANTAFÉ DE BOGOTÁ. Plan de desarrollo Económico, social y de obras públicas para Bogotá.1998- 2001.Acuerdo 6 de 1998. Bogotá, 1998.

ARDILA GOMEZ, Arturo. Gestión Local y Descentralización. Alcaldía Mayor de Bogotá, Secretaria de Gobierno, Subsecretaría de Asuntos Locales. Bogotá,1997.

BOTERO ZEA, Fernando. Bogotá Descentralizada. Editorial Uniandes. Bogotá, 1990.

CONTRALORIA DE SANTAFÉ DE BOGOTÁ D.C. Informe de Ejecución Presupuestal del Distrito Especial de Bogotá 1988. Bogotá,1989.

CONTRALORIA DE SANTAFÉ DE BOGOTÁ D.C. Informe de Ejecución Presupuestal del Distrito Especial de Bogotá 1992. Bogotá,1993.

CONTRALORIA DE SANTAFÉ DE BOGOTÁ D.C. Informe de Ejecución Presupuestal del Distrito Especial de Bogotá 1993. Bogotá,1994.

CONTRALORIA DE SANTAFÉ DE BOGOTÁ D.C. Informe Financiero de Santafé de Bogotá A diciembre 31 de 1994.Bogotá,1995.

CONTRALORIA DE SANTAFÉ DE BOGOTÁ D.C. Informe Financiero de Santafé de Bogotá A diciembre 31 de 1995. Bogotá, 1996.

CONTRALORIA DE SANTAFÉ DE BOGOTÁ D.C. Informe Financiero de Santafé de Bogotá A diciembre 31 de 1996. Bogotá, 1997.

CONTRALORIA DE SANTAFÉ DE BOGOTÁ D.C. Informe Financiero de Santafé de Bogotá A diciembre 31 de 1997. Bogotá, 1998.

CONTRALORIA DE SANTAFÉ DE BOGOTÁ D.C. Informe Financiero de Santafé de Bogotá A diciembre 31 de 1998. Bogotá, 1999.

42 Teodomiro Muñoz Criollo 
CONTRALORIA DE SANTAFÉ DE BOGOTÁ D.C. Informe Financiero de Santafé de Bogotá A diciembre 31 de 1999. Bogotá, 2000.

CONTRALORIA DE SANTAFÉ DE BOGOTÁ D.C. Evaluación de Gestión de las localidades del Distrito Capital 1993 - 1994. Bogotá, 1995.

CONTRALORIA DE SANTAFÉ DE BOGOTÁ D.C. Evaluación de Gestión de las localidades del Distrito Capital 1995. Bogotá, 1996.

CONSTITUCIÓN POLÍTICA DE COLOMBIA 1991. Imprenta Nacional. Bogotá, 1992.

DEPARTAMENTO NACIONAL DE ESTADISTICAS. DANE. Guía para el Uso del I.P.C. Bogotá, 1998.

DEPARTAMENTO ADMINISTRATIVO DE PLANEACIÓN DISTRITAL. Estadísticas de Santa fe de Bogotá D.C., 1997.

ESTATUTO ORGANICO DE SANTA FE DE BOGOTA DISTRITO CAPITAL. Decreto ley 1421 de 1993. Imprenta Nacional, 1999.

GONZALEZ GALVEZ, Beatriz Eugenia. Laberinto Descentralista. Pontificia Universidad Javeriana. Bogotá, 1990.

JUNTAS ADMINISTRADORAS LOCALES DE SANTAFÉ DE BOGOTÁ. D.C. Planes de Desarrollo Locales 1996 - 1998. Bogotá, 1996.

JUNTAS ADMINISTRADORAS LOCALES DE SANTAFÉ DE BOGOTÁ. D.C. Planes de Desarrollo Locales 1999 - 2001. Bogotá, 1999.

SALDIAS BARRENECHE, Carmenza. Como van las finanzas Distritales en Bogotá. Cómo vamos? Facultad de Ingeniería de la Universidad de los Andes. Bogotá, 1999.

SARMIENTO PALACIO, Eduardo. La situación Financiera del Distrito Capital en las Finanzas del Distrito Capital. Cámara de Comercio, FESCOL. Bogotá, 1995. 
